ed evidence that T-cell receptor rearrangement is exceedingly rare in $\mathrm{B}$ cells and at the same time reject the even more widely accepted evidence that $\mathrm{T}$ cells do not hypermutate their T-cell receptor loci.

\section{Jürgen Bach}

Matthias Wabl

Department of Microbiology and Immunology,

University of California at San Francisco,

San Francisco,

California 94143-0670, USA

KeLSOE ET AL. REPLY - Bachl and Wabl are mistaken in stating that we presented insufficient data for a proper analysis of our results. Mutations in $\mathrm{V} \alpha$ genes arise in vivo as a consequence of reaction in the germinal centre (GC) or in vitro during the PCR. Both processes are subject to Luria-Delbrück fluctuations and, in fact, the mutations observed in GCs fit a Luria-Delbrück distribution. However, the in vivo process is resistant to quantification since many factors are unkown (for example, replicative rates, independence of samples, effects of selection). In contrast, mutations introduced by the PCR can be rigorously analysed. Therefore, the null hypothesis that mutations in all samples were introduced in vitro can be tested.

We used a Monte Carlo algorithm to estimate the spectrum of PCR errors, including 'jackpot effects' due to early introduction of misincorporations, as follows. At each round of a simulated PCR, templates replicate with probability $\varepsilon$ $(0.8)$. If successful, each daughter independently incorporates a number of new mutations with parameter $\mu$ given by the product of the error rate of the Pfu polymerase and the length of the template

sequence $^{1}$; this is repeated for 80 cycles. This simulation was repeated $10^{4}$ times to estimate the median, mean, $99 \%$ and $99.9 \%$ quantiles for frequencies of $\mathrm{V} \alpha 11 / \mathrm{J} \alpha 11$ rearrangements containing 1 , 2 or $\geqslant 3$ PCR errors. Predicted frequencies did not differ significantly from those observed in the B10 cell line (1 mutation observed versus 1.7 expected) and in periarteriolar T-cell sheath (PALS) cells (7 observed versus 4.2 expected). In contrast, $\mathrm{V} \alpha 11$ mutations in GC cells were significantly in excess of expected PCR mistakes; for example, the number of $V \alpha$ exons with three mutations was about 3,500-fold above that expected. By chance, $<0.01 \%$ of sampled populations would contain triply mutated sequences at frequencies $\geqslant 1 / 4,500$; the frequency we observed for triple mutants in GCs was approximately $1 / 57$.

Alternatively, problems of fluctuation may be avoided simply by scoring repeated mutations as single events. This analysis shows mutations in GCs to be significantly greater $(P=0.03$; Fisher's exact test) than that found in the PALS.

Bachl and Wabl correctly note that mutated $V \alpha$ rearrangements might originate in B lymphocytes. However, our present studies of single $\mathrm{T}$ cells dissected from GCs demonstrate V $\alpha 11$ mutants in about $10 \%$ of productive rearrangements. Garnett Kelsoe, Biao Zheng

Department of Microbiology and Immunology,

University of Maryland School of Medicine,

Baltimore, Maryland 21201, USA

Thomas B. Kepler

Biomathematics Program, Department of Statistics,

North Carolina State University, Raleigh, North Carolina 27695, USA

\title{
Redshift and black-hole mass
}

SIR - The very interesting observations by M. Myoshi et al. (Nature 373, 127-129; 1995) of water-vapour maser emission in the NGC 4258 disk offer an additional direct test for their model of a high-rotation velocity disk and allow an independent determination of the central blackhole mass. Indeed, a detectable amount of gravitational redshift should affect the observed line wavelengths.

As the distances of the maser emission regions from the centre of the black hole $r$ are much larger than the black-hole Schwarzschild radius $r_{\mathrm{S}}=2 G M / c^{2}=3.5$ $10^{-6} \mathrm{pc}$, the gravitational redshift is simply given by (assuming a spherical symmetry):

$$
z(r)=\frac{r_{\mathrm{S}}}{2 r}=\frac{v_{\text {circ }}^{2}}{c^{2}}
$$

where $G$ is the gravitational constant, $M$ the mass of the central object, $v_{\text {circ }}$ the velocity of a particle in keplerian motion around it at radius $r$ and $c$ the velocity of light. The gravitational redshift expected for a central mass of $3.6 \times 10^{7} M_{\odot}$ at the inner edge of the disk is $c z(r=0.13 \mathrm{pc})=$ $4.0 \mathrm{~km} \mathrm{~s}^{-1}$, whereas the one at the outer edge is $c z(r=0.26 \mathrm{pc})=2.0 \mathrm{~km} \mathrm{~s}^{-1}$, more than 10 times their velocity resolution, or about 4 and 2 times their quoted errors on the velocity determination for the highvelocity lines.

The high-rotation velocity lines offer an easy and safe way to test the disk model and to determine the black-hole mass. The reason is that they apparently arise very close to a line perpendicular to the line-of-sight. As a consequence, their velocities should systematically deviate from the values expected from keplerian motion, with residuals that are inversely proportional to the distances of the maser emission regions from the centre of the black hole. Further, the keplerian motion velocities have an antisymmetrical behaviour relative to the centre of the disk, whereas the gravitational redshifts have a symmetrical one. The low-velocity lines should also be affected by a gravitational redshift of $4.0 \mathrm{~km} \mathrm{~s}^{-1}$ as they are produced at the inner edge of the disk $(r=0.13 \mathrm{pc})$.

Alain Smette

Konrad Kuijken

Kapteyn Astronomical Institute,

PO Box 800 ,

NL-9700 AV Groningen, The Netherlands

\section{Collision age}

SIR - Beck et al. ${ }^{1}$ have reported new biogeographical data, concluding that the collision of the Indian subcontinent with Asia was older than previously claimed. They suggested that the collision occurred between 66 and 55.5 Myr (million years) ago. However, palaeontological data constrain the latest possible date even more accurately. From the study of continental faunas and floras, it has been known for several years that terrestrial continuity between India and mainland Asia was already established by the time of the K/T boundary, $65 \mathrm{Myr}$ ago, or probably slightly earlier ${ }^{2,3}$. More recent data again support this view ${ }^{4,5}$.

Nevertheless, an Eocene collision, which was accepted for a long time, appears still to be admitted by various workers. The biostratigraphical result of Beck et al. is an additional argument which demonstrates that such an age can no be longer accepted.

J.-C. Rage

Laboratoire de paléontologie des vertébrés,

Université Paris VI, case 106 , 75252 Paris cedex 05, France

\section{H. Cappetta}

J.-L. Hartenberger

J.-J. Jaeger

J. Sudre

\section{Vlaney-Liaud}

Institut des Sciences de I'Evolution, Université de Montpellier II, case 64, Place Eugène Bataillon.

34095 Montpellier cedex 5, France K. Kumar

Wadia Institute of Himalayan Geology, 33 General Mahadeo Singh Road, Dehra Dun 248001, India

\section{G. V. R. Prasad}

PG Department of Geology,

University of Jammu,

Jammu Tawi 180004, India

A. Sahni

Center of Advanced Geological Studies,

Panjab University,

Chandigarh 160014, India

1. Beck, R. A. et al. Nature 373, 55-58 (1995).

2. Sahni, A. et al. Bull. Soc. géol. Fr. 14, 1093-1099 (1982).

3. Hartenberger, J.-L. Bull. Soc. Palaeont. Ital. 21, 283-288 (1982).

4. Prasad, G. V. R. et al. J. Vertebr. Paleont. 14, 260-277 (1994).

5. Prasad, G. V. R. \& Rage, J.C. Cretaceous. Res. 16, 95-107 (1995). 